\title{
Strong lensing efficiency of galaxy clusters in dark energy cosmologies
}

\author{
M. Meneghetti ${ }^{1}$, M. Bartelmann ${ }^{1}$, K. Dolag ${ }^{2,7}$, L. Moscardini ${ }^{3}$, F. Perrotta ${ }^{4,5,6}$, C. Baccigalupi ${ }^{4,5,6}$, and G. Tormen ${ }^{2}$ \\ 1 Zentrum für Astronomie der Universität Heidelberg, ITA, Albert-Überle-Str. 2, 69120 Heidelberg, Germany \\ e-mail: massimo.meneghetti@ita.uni-heidelberg.de \\ 2 Dipartimento di Astronomia, Università di Padova, Vicolo dell'Osservatorio 2, 35120 Padova, Italy \\ 3 Dipartimento di Astronomia, Università di Bologna, via Ranzani 1, 40127 Bologna, Italy \\ 4 SISSA/ISAS, via Beirut 4, 34014 Trieste, Italy \\ 5 INFN, Sezione di Trieste, via Valerio 2, 34127 Trieste, Italy \\ 6 Lawrence Berkeley National Laboratory, 1 Cyclotron Road, Berkeley CA 94720, USA \\ 7 Max-Planck-Institut für Teoretische Astrophysik, 85748 Garching, Germany
}

Received 5 May 2004 / Accepted 11 June 2005

\section{ABSTRACT}

We study the efficiency for producing strong gravitational lensing events of galaxy clusters numerically simulated in different dark-energy cosmologies with constant and time-variable equation of state, and we compare the results with those obtained in "standard" $\Lambda C D M$ and OCDM models. Our main results are (1) that the expected abundance of gravitational arcs with large length-to-width ratio depends on the equation of state of dark energy at the epoch of formation of the halo; and (2) that the high sensitivity of strong-lensing cross sections of galaxy clusters to dynamical processes like mergers, which was found in earlier studies, varies substantially between different cosmologies, being stronger for models in which halos are less concentrated. As expected, the largest differences in the lensing optical depth occur at intermediate and high redshift.

Key words. gravitational lensing - cosmology: theory - galaxies: clusters: general

\section{Introduction}

Evidence is mounting that the Universe is spatially flat, has low matter density and is dominated by some form of dark energy, acting as a repulsive gravitational force and being responsible for the present phase of accelerated cosmic expansion (e.g. Riess et al. 1998; Perlmutter et al. 1999; Hawkins et al. 2003; Spergel et al. 2003; Tegmark et al. 2004; Riess et al. 2004). Dark energy generalises the concept of a cosmological constant, admitting dynamics and fluctuations. Such a generalisation is necessary attempting to reconcile the dark-energy density with the known energy scales of particle physics. The minimal extension of a cosmological constant is a self-interacting scalar field (quintessence, see Peebles \& Ratra 2002, and references therein), which was already considered well before the evidence for cosmic acceleration (Wetterich 1988; Ratra \& Peebles 1988).

Structures tend to grow earlier in dark-energy than in cosmological-constant models, which renders dark-matter haloes more concentrated (Bartelmann et al. 2002; Weinberg \& Kamionkowski 2003; Dolag et al. 2004). Following earlier analytic work (Bartelmann et al. 2003), we study here with cluster models numerically simulated in eight different cosmologies how earlier structure growth and denser halo cores affect strong lensing by galaxy clusters. Our motivation is three-fold. First, despite recent attempts (Oguri et al. 2003; Wambsganss et al. 2004; Dalal et al. 2004), it remains to be clarified whether the strong-lensing efficiency of real galaxy clusters is reproduced by numerical cluster models or not. Earlier studies found that the expected strong-lensing efficiency of galaxy clusters in cosmological-constant models appears to fall short by an order of magnitude of reproducing the observed number of arcs (Bartelmann et al. 1998). We thus wish to quantify the effect on the strong-lensing optical depth of the increased halo core density in dark-energy models. Second, earlier structure growth may have interesting implications for strong cluster lensing in view of the recently-detected clusters at redshifts near unity in which strongly-lensed arcs were found (Hasinger et al. 1998; Thompson et al. 2001; Gladders et al. 2003; Zaritsky \& Gonzalez 2003; Lidman et al. 2004). Third, it has been found in a recent study with high time resolution (Torri et al. 2004) that the efficiency of a cluster for strong lensing can substantially be increased during merger processes because of the enhanced tidal field and surface-mass 
density during different phases of the merger process. Shifting structure growth to earlier redshifts also allows major mergers at higher redshifts and thus potentially much increased arcformation probabilities. Of course, a comparison between simulations and observations is still premature, due to both the lack of strong observational constraints and the poor knowledge of the connection between strong-lensing efficiency and clustermerger dynamics. Nonetheless, we aim at analysing and understanding the principal effects of dynamical dark energy on strong lensing by clusters. This numerical study complements and extends our analytic work (Bartelmann et al. 2003) towards realistic, asymmetric, substructured and evolving mass distributions.

With these motivations in mind, we use the 17 cluster-sized dark matter haloes described in Dolag et al. (2004) and investigate their strong-lensing properties. Section 2 describes the eight cosmological models used, Sect. 3 the cluster sample, and Sect. 4 the lensing simulations. Results are presented in Sect. 5 and summarised in Sect. 6.

\section{Cosmological models}

We compare in this paper the efficiency for producing strong lensing events of the sample of numerically simulated clusters previously described in Dolag et al. (2004). We give here a short description of both the cosmological models and the methods used for obtaining the cluster sample, and refer the reader to our earlier paper for further detail.

All clusters in the sample were simulated in different cosmologies with constant and time-variable equations of state. These are an open Cold Dark Matter (OCDM) and four spatially flat cosmological models, namely a cosmologicalconstant $(\Lambda \mathrm{CDM})$ model, a dark-energy model with constant equation of state (DECDM), and two quintessence models with markedly different dynamical properties, one with inverse power-law Ratra-Peebles potential (RP, see Peebles \& Ratra 2002, and references therein) and one with SUGRA potential (SUGRA, see Brax \& Martin 2000, and references therein). For the latter three, we have used two different normalisations of the perturbation power spectrum, to be discussed later.

In all cases, the matter density parameter today is $\Omega_{0}=0.3$. In the flat cosmologies, the remaining $70 \%$ of the critical density are assigned to the dark energy at present, thus $\Omega_{\Lambda}=0.7$. The remaining cosmological parameters are $h=0.7, \Omega_{\mathrm{b}} h^{2}=0.022$, Gaussian density fluctuations with scale-invariant power spectrum, and no gravitational waves.

The normalisation of the perturbation power spectrum, which we set conventionally through the rms density fluctuation level $\sigma_{8}$ within spheres of $8 h^{-1} \mathrm{Mpc}$ radius, has been chosen as $\sigma_{8}=0.9$ for all the cosmological models in order to match the observed abundance of galaxy clusters irrespective of the model. Since the power-spectrum normalisation is most important here, affecting the structure statistics and thus the lensing power, we alternatively normalise the DECDM, RP and SUGRA models to the observed level of anisotropies in the Cosmic Microwave Background (CMB) (e.g. Bennett et al. 2003). In this case, the amplitude $\sigma_{8}$ is generally slightly smaller because of the Integrated Sachs Wolfe (ISW) effect affecting the large scale CMB anisotropies: the time interval is longer compared to the cosmological-constant case in which the cosmic equation of state changes in dark-energy scenarios. This enhances the dynamics of the gravitational potentials and increases the ISW power, which is visible in the wideangle tail of CMB anisotropy power spectrum (see Bartelmann et al. 2002, and references therein). We take $\sigma_{8}=0.86,0.82$ and 0.76, respectively, for DECDM, RP and SUGRA. Note that the reduction is quite important, since we selected our darkenergy models in order to highlight the differences between them and the cosmological-constant model. If the equation-ofstate parameter is smaller than in the cases we consider here, the ISW effect and the reduction to the power-spectrum normalisation are also smaller.

These numbers, as well as all the inputs from the linear evolution of cosmological perturbations to the $N$-body procedure described later, are computed using our dark-energy oriented cosmological code, based on CMBfast (Seljak \& Zaldarriaga 1996). The code, originally introduced by Perrotta \& Baccigalupi (1999), describes the dark energy as a scalar field, numerically integrating its background and linear perturbation dynamics for the most relevant tracking quintessence scenarios, capable of converging to the present phase of cosmic acceleration from a wide range of initial conditions in the early universe (Baccigalupi et al. 2000). This is performed in the general framework of scalar-tensor cosmologies, in which the dark energy may be directly linked with gravity (Perrotta et al. 2000). The output of this machinery has been used for other work, allowing us in particular to constrain the darkenergy equation of state with the modern CMB anisotropy observations (Baccigalupi et al. 2002).

The dark energy cosmologies are characterised by their equation of state parameter $w(z)$, describing the ratio between pressure and energy density of the dark energy. For cosmic expansion to be accelerated today, the $w$ parameter must be $<-1 / 3$. In the limit of constant $w$, the dark-energy density evolves with redshift in proportion to $(1+z)^{3(1+w)}$. This applies to both the $\Lambda$ CDM model, whose $w=-1$ yields a constant dark energy density, and the DECDM model, which we choose to have $w=-0.6$. It is useful to note that from the point of view of the Friedmann equation, the curvature term in the OCDM model behaves as a dark energy component with constant $w=-1 / 3$ (see Bartelmann et al. 2002, and references therein).

For the RP and SUGRA models, $w$ is time-dependent. The dark energy is consistently described by means of the quintessence scalar field $\phi$. The RP and SUGRA quintessence potentials are given by

$$
V_{\mathrm{RP}}=\frac{M^{4+\alpha_{Q}}}{\phi^{\alpha_{Q}}}, \quad V_{\mathrm{SUGRA}}=\frac{M^{4+\alpha_{Q}}}{\phi^{\alpha_{Q}}} \exp \left(4 \pi G \phi^{2}\right),
$$

respectively. The exponential in $V_{\text {SUGRA }}$ reflects super-gravity corrections (Brax \& Martin 2000) and induces large variations in the equation of state at the epoch of structure formation compared to the RP case, even if $w$ is the same at present in the two models. Dolag et al. (2004) found indeed that the cluster concentration reflects the dark-energy behaviour at the epoch of halo collapse, a promising feature in order to reduce 


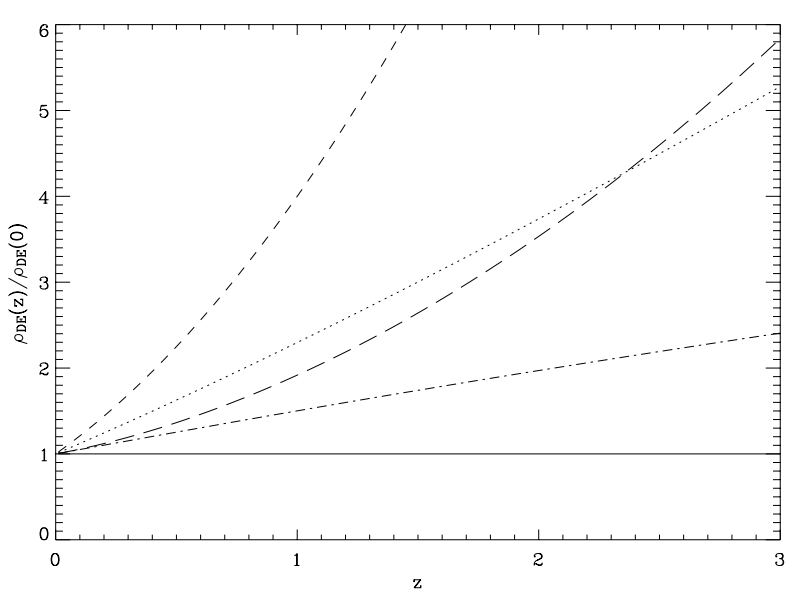

Fig. 1. Energy density vs. $z$ for the $\Lambda$ CDM model (solid line), and the RP (dot-dashed), SUGRA (long-dashed), DECDM (dotted) and OCDM (short-dashed) models analysed in this paper.

Table 1. Cosmological parameters used in the different simulation runs.

\begin{tabular}{ccc}
\hline \hline Model & $\sigma_{8}$ & $w_{0}$ \\
\hline OCDM & 0.9 & 0 \\
$\Lambda$ CDM & 0.9 & -1 \\
SUGRA & $0.76(0.9)$ & -0.83 \\
RP & $0.82(0.9)$ & -0.83 \\
DECDM & $0.86(0.9)$ & -0.6 \\
\hline
\end{tabular}

the degeneracy between different dark-energy models yielding the same amount of acceleration today. In this work, we study how this behaviour affects the statistics of strongly lensed arcs in clusters. Specifically, the tracking RP and SUGRA quintessence models considered here have $w_{0}=-0.83$ today, with $\alpha_{Q}=-0.6$ and $\alpha_{Q}=-6.7$, respectively. In the tracking regime, this yields $w_{\text {SUGRA }} \simeq-0.23$ and $w_{\text {RP }} \simeq-0.77$.

Summarising the differences between the various cosmologies, we show in Fig. 1 the energy density as a function of redshift for the different models used in the paper. A list of the cosmological parameters used for each run is given in Table 1 .

\section{Cluster sample}

The cluster models used in this study were simulated using a new version of the cosmological code GADGET (Springel et al. 2001), specifically adapted to simulate the formation and evolution of cosmic structures in the dark energy models described earlier (see Dolag et al. 2004).

Each cluster in our sample was obtained using the socalled re-simulation technique, which consists in re-simulating at higher resolution a patch of a pre-existing large-scale cosmological simulation (Tormen et al. 1997). For this work, we selected as a parent simulation an $N$-body run with $512^{3}$ particles in a box of $479 h^{-1} \mathrm{kpc}$ (Yoshida et al. 2001; Jenkins et al. 2001). Its background cosmological model is spatially flat with $\Omega_{\mathrm{m}, 0}=0.3$ and $\Omega_{\Lambda}=0.7$ at the final epoch, identified with redshift zero. The particle mass was $6.82 \times 10^{10} h^{-1} M_{\odot}$, and the gravitational softening was chosen as $30 \mathrm{~h}^{-1} \mathrm{kpc}$. From the output of this simulation at $z=0$, we selected ten spherical regions of radius between 5 and $10 h^{-1} \mathrm{Mpc}$, each containing either one or a pair of dark-matter haloes with mass exceeding $3 \times 10^{14} h^{-1} M_{\odot}$. We thus obtain a sample of 17 cluster-sized objects.

Each of these regions was re-sampled to build new initial conditions with on average $10^{6}$ dark-matter particles. The initial conditions from the $\Lambda \mathrm{CDM}$ cosmology were adapted to all the dark energy cosmologies studied here as described in Dolag et al. (2004). This was done in two steps. In the first step, the initial redshift was adapted such that the rms density fluctuation amplitude reached the desired normalisation amplitude $\sigma_{8}$ today, despite the different dynamics of the models. In a second step, the peculiar velocities of the particles in their Lagrangian initial region were modified such as to reflect the change in initial redshift. The mass resolution of the re-simulation ranged from $2 \times 10^{9}$ to $6 \times 10^{9} \mathrm{~h}^{-1} M_{\odot}$ per dark-matter particle so as to have each cluster consisting of approximately the same number of particles. The gravitational softening was reduced to a $5 h^{-1} \mathrm{kpc}$ cubic-spline smoothing for all particles in the highresolution region.

We found that our 17 clusters contain on average $N_{V} \approx 200000$ dark matter particles within their virial radii. The corresponding virial masses range from $M_{V}=3.1 \times 10^{14}$ to $1.7 \times 10^{15} h^{-1} M_{\odot}$.

\section{Lensing simulations}

Ray-tracing simulations are then carried out with each of the 17 clusters in all eight cosmological models. The technique used in this study was described in detail in several earlier papers (e.g. Bartelmann et al. 1998; Meneghetti et al. 2000).

For all clusters in each cosmological model, we produce $N_{\text {snap }}=52$ snapshots at different redshifts between zero and unity. These are equispaced in time, leading to a time resolution of $\sim 100 \mathrm{Myr}$, which allows the effects of dynamical processes on the cluster cross sections for strong lensing to be resolved. This is important given the rapid and substantial changes in strong-lensing cross sections during mergers, which were found and discussed in Torri et al. (2004).

For each snapshot, we select a cube of $3 h^{-1}$ Mpc comoving side length, centred on the halo centre and containing the highdensity region of the cluster. The particles in this cube are used for producing a three-dimensional density field, by interpolating their position on a grid of $256^{3}$ cells using the Triangular Shaped Cloud method (Hockney \& Eastwood 1988). Then, we project the three-dimensional density field along the coordinate axes, obtaining three surface density maps $\Sigma_{i, j}$ per snapshot, used as lens planes in the following lensing simulations. The total number of lensing simulations is therefore $17 \times 52 \times 3 \times 8=21216$, requiring approximately the same number of computation hours on an IBM-SP4 cluster located at the computer centre of the Max Planck Society in Garching, Germany.

The lensing simulations are performed by tracing a bundle of $2048 \times 2048$ light rays through a regular grid, covering the central quarter of the lens plane. This choice is driven by the necessity of studying in detail the central regions of the clusters, where critical curves form, taking into account the contribution 
from the surrounding mass distribution to the deflection angle of each ray.

Deflection angles on the ray grid are computed using the method described in Meneghetti et al. (2000). We first define a grid of $128 \times 128$ "test" rays, for each of which the deflection angle is calculated by directly summing the contributions from all cells on the surface density map $\Sigma_{i, j}$,

$\alpha_{h, k}=\frac{4 G}{c^{2}} \sum_{i, j} \Sigma_{i, j} A \frac{\boldsymbol{x}_{h, k}-\boldsymbol{x}_{i, j}}{\left|\boldsymbol{x}_{h, k}-\boldsymbol{x}_{i, j}\right|^{2}}$,

where $A$ is the area of one pixel on the surface density map and $\boldsymbol{x}_{h, k}$ and $\boldsymbol{x}_{i, j}$ are the positions on the lens plane of the "test" ray $(h, k)$ and of the surface density element $(i, j)$. Following Wambsganss et al. (1998), we avoid the divergence at zero distance between a light ray and the density grid-point by shifting the "test" ray grid by half-cells in both directions with respect to the grid on which the surface density is given. We then determine the deflection angle of each of the $2048 \times 2048$ light rays by bi-cubic interpolation between the four nearest test rays.

The position $\boldsymbol{y}$ of each ray on the source plane is calculated using the lens equation. If $\boldsymbol{y}$ and $\boldsymbol{x}$ are the angular positions of source and image from an arbitrarily defined optical axis passing through the observer and perpendicular to the lens and source planes, this is written as

$y=x-\frac{D_{\mathrm{ls}}}{D_{\mathrm{s}}} \alpha(x)$

where $D_{\mathrm{ls}}$ and $D_{\mathrm{s}}$ are the angular-diameter distances between the lens and the source planes, and between the observer and the source plane, respectively.

Then, a large number of sources is distributed on the source plane. We place this plane at redshift $z_{\mathrm{s}}=1$. Keeping all sources at the same redshift is an approximation justified for the purposes of the present case study, but the recent detections of arcs in high-redshift clusters (Hasinger et al. 1998; Thompson et al. 2001; Gladders et al. 2003; Zaritsky \& Gonzalez 2003; Lidman et al. 2004) indicate that more detailed simulations will have to account for a wide source redshift distribution.

The sources are elliptical with axis ratios randomly drawn with equal probability from $[0.5,1]$. Their equivalent diameter (the diameter of the circle enclosing the same area of the source) is $r_{\mathrm{e}}=1^{\prime \prime}$. They are distributed over a region on the source plane corresponding to one quarter of the field of view where rays are traced. As in our earlier studies, we adopt an adaptive refinement technique when placing sources on their plane. We first start with a coarse distribution of $32 \times 32$ sources and then increase the source number density towards the high-magnification regions of the source plane by adding sources on sub-grids whose resolution is increased towards the lens caustics. This increases the probability of producing long arcs and thus the numerical efficiency of the method. In order to compensate for this artificial source-density enhancement, we assign a statistical weight to each image for the following statistical analysis which is proportional to the area of the sub-grid cell on which the source was placed.

By collecting rays whose positions on the source plane fall within any single source, we reconstruct the images of background galaxies and measure their length and width. Our technique for image detection and classification was described in detail by Bartelmann \& Weiss (1994) and adopted by Bartelmann et al. (1998); Meneghetti et al. (2000, 2001, 2003a,b) and Torri et al. (2004). It results in a catalogue of simulated images which is subsequently analysed statistically.

\section{Results}

In this section, we discuss how the lensing properties of the clusters in our sample differ in the different cosmological models.

\subsection{Critical curves and caustics}

We start by considering the critical curves, along which strongly magnified and highly distorted images form. These are the lines on the lens plane where the determinant of the Jacobian matrix of the lens mapping vanishes,

$A_{h k}(\boldsymbol{x}) \equiv \frac{\partial y_{h}}{\partial x_{k}}=\delta_{h k}-\frac{\partial \alpha_{h}}{\partial x_{k}}=0$.

Since the local magnification is the inverse of the Jacobian determinant, the critical points are ideally characterised by infinite magnification.

Through the lens equation, critical lines are mapped onto the caustics in the source plane. Sources lying close to the caustics therefore have images strongly magnified in the radial or tangential directions, i.e. they are imaged as gravitational arcs. The probability of one cluster to produce highly distorted images is thus closely related to the size and the shape of the critical curves and of the caustics.

We show in Fig. 2 some examples of how the critical curves (and of the corresponding caustics) of one of the clusters in our sample change between different cosmological models. The halo has a virial mass ranging from $4.3 \times 10^{14} h^{-1} M_{\odot}$ to $5.2 \times 10^{14} h^{-1} M_{\odot}$ at redshift $z \sim 0.3$. Considering models characterised by the same normalisation of the power spectrum of the primordial density perturbations, it is clearly seen that the two models with dynamical dark energy, i.e. the RP and SUGRA models, and the DECDM model with constant equation of state $w=-0.6$, interpolate between the $\Lambda \mathrm{CDM}$ and the OCDM model. In particular, the critical curves in the SUGRA model have sizes comparable to those in the DECDM model.

However, it is important to note that the size of the critical curves and of the caustics in dark energy models may be smaller when the CMB normalisation is used, because of the enhanced ISW effect with respect to the $\Lambda$ CDM model. The larger the required reduction of $\sigma_{8}$ is, the more the critical curves shrink; in the case considered here, the critical curves tend to have sizes comparable to those in the $\Lambda \mathrm{CDM}$ model or smaller.

\subsection{Lensing cross sections}

Earlier work found the length-to-width ratio of gravitational arcs to be very sensitive to many intrinsic properties of the 
M. Meneghetti et al.: Strong lensing efficiency of galaxy clusters in dark energy cosmologies

\begin{tabular}{|c|c|c|c|c|c|c|c|}
\hline 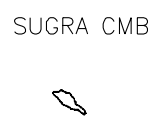 & $\begin{array}{l}\text { RP CMB } \\
8\end{array}$ & $\Lambda C D M$ & st & DECDM CMB & SUGRA & $S^{\mathrm{DECDM}}$ & OCDM \\
\hline 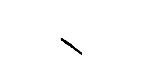 & ' & ' & ' & $\$$ & $\nabla$ & $\Downarrow$ & $\gamma$ \\
\hline
\end{tabular}

Fig. 2. Critical curves (upper panels) and caustics (bottom panels) of one of the clusters in our sample in all eight cosmological models. The side length of each panel is $50^{\prime \prime}$.

lenses which depend on cosmology (Bartelmann \& Weiss 1994; Bartelmann et al. 1995, 1998). Modelling clusters using the NFW density profile, which depends on cosmology in contrast to the singular isothermal profile, such a high sensitivity of the abundance of long and thin arcs on cosmology was confirmed even using an analytic approach (Meneghetti et al. 2003b; Bartelmann et al. 2003).

Thus, aiming at evaluating the differences between the strong-lensing efficiency of clusters in different cosmological models with dark energy, we focus on the statistical distributions of the length-to-width ratios of the simulated gravitational arcs.

The efficiency of one galaxy cluster for producing arcs with a given property can be quantified by means of its lensing cross section. By definition, this is the area on the source plane where a source must be placed in order to be imaged as an arc with that property. As explained in the previous sections, we artificially increase the density of background sources close to the cluster caustics by adopting adaptive grid refinement. Each source is taken to represent a fraction of the source plane. We assign to each source and all of its images a statistical weight $w$ which is inversely proportional to the squared resolution of the sub-grid on which the corresponding source was placed. The cells of the sub-grid with the highest resolution have area $A$, and the sources placed on its grid points are given a statistical weight of unity. The absolute lensing cross sections are then determined by counting the statistical weights of the sources whose images have a length-to-width ratio exceeding a threshold $(L / W)_{\min }$. If a source has multiple images with $(L / W) \geq(L / W)_{\min }$, we multiply its statistical weight by the number of such images. Accordingly, the lensing cross section is

$\sigma_{(L / W)_{\min }}=A \sum_{i} W_{i} w_{i} n_{i}$

where $W_{i}$ is unity if the $i$ th source has images with $(L / W) \geq$ $(L / W)_{\min }$ and zero otherwise, $n_{i}$ is the number of images of the $i$ th source satisfying the required condition, and $w_{i}$ is the statistical weight of the source.

\subsubsection{Averaged cross sections}

We now discuss the averaged cross sections of our cluster sample. The lensing cross sections vary significantly among halos

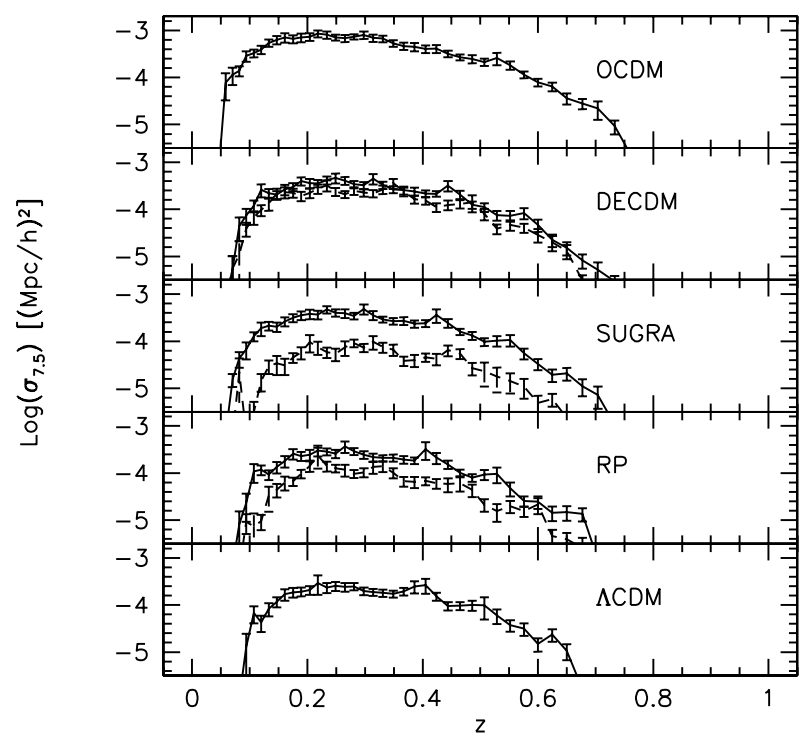

Fig. 3. Averaged lensing cross sections for arcs with length-to-width ratio larger than 7.5 of our cluster sample as functions of the lens redshift. Sources are kept at redshift $z_{\mathrm{s}}=1$. Different panels show the results for different cosmological models. Solid and dashed curves, respectively, display averaged cross sections in cosmological models with $\sigma_{8}=0.9$ and with $\sigma_{8}$ lowered for taking into account the increasing ISW effect affecting the large angular scales in the CMB fluctuations. Attached to all curves are bootstrap error bars illustrating the uncertainty of the arc cross sections.

of different mass and dynamical state, thus we aim at the overall strong-lensing statistics rather than the lensing properties of individual clusters.

In Fig. 3 we show the lensing cross sections for long and thin arcs, averaged over all 17 clusters, as function of the lens redshift for all eight cosmological models. For each cluster, we use its three projections along the coordinate axes for measuring the cross section. Therefore, the curves displayed in Fig. 3 result from averaging over 51 different curves for each cosmological model.

Confirming expectations from our analytic studies, the lensing cross sections reflect the differences in the concentration of dark matter halos in different cosmological models. This has been also discussed in our previous paper (Dolag et al. 2004). It is shown there that the cluster concentration depends on the dark energy equation of state at the cluster 
formation redshift $z_{\text {coll }}$ through the linear growth factor $D_{+}\left(z_{\text {coll }}\right)$. Assuming the same normalisation of the power spectrum, the lensing cross sections for the OCDM and the $\Lambda$ CDM models differ by roughly a factor of four, independent of the minimal length-to-width ratio of the arcs, and the cross sections for the other cosmological models with dark energy interpolate between them. Despite the present-time equation of state parameter of the dark energy being the same in the RP and SUGRA models, their lensing cross sections differ substantially at higher redshift. Similar results were found in a recent independent work by Macciò (2005) for cosmological models characterized by different dynamical dark energy parameters.

For models with the CMB normalisation of the power spectrum, we find lensing cross sections smaller by even more than one order of magnitude compared to the OCDM model. In fact, when the normalisation is reduced because of the ISW effect affecting the large-scale CMB anisotropies in the cosmologies we consider (Bartelmann et al. 2003), not only the formation epoch of our simulated clusters is delayed, but also their evolution up to redshift zero is changed. For example, clusters in the RP and in the SUGRA models have masses at redshift zero which are smaller by roughly $20 \%$ and $30 \%$, respectively, compared to the $\Lambda \mathrm{CDM}$ model.

We perform a bootstrap analysis of the rms uncertainty of the averaged arc cross sections. The bootstrap error bars are shown in each panel. At each redshift we bootstrapped the lensing cross sections corresponding to the $17 \times 3$ lens planes. We found the bootstrap errors to be at most $25 \%$ despite the large range of masses of the clusters in our sample. This shows that, although the cluster sample is not large, the sample variance does not strongly affect our results.

The cluster sample is still too small for the averaged cross sections to be smooth functions of cluster redshift. In fact, the curves exhibit pronounced peaks which are caused by merger events, i.e. the infall of secondary clumps of matter into the virial regions of the clusters. It has recently been shown that during such events, on timescales of some hundred Myr, the efficiency for strong lensing by clusters is sharply and strongly enhanced (Torri et al. 2004), due to the combined effects of the increasing shear and convergence. In principle, such sharp peaks in the strong-lensing efficiency might also be produced by clumps of matter orbiting outside the virial region of the cluster but passing very close to the cluster core when their motion is projected along the line of sight. However, their impact on arc statistics is expected to be significantly smaller because if they orbit at large distances from the cluster centre, they will most likely enhance the lensing cross section only in one of the three cluster projections. We will discuss the properties of these peaks in the next subsection, when the lensing cross sections of one individual cluster will be shown.

\subsubsection{Individual clusters}

In Fig. 3, several peaks can be found at least in all the curves obtained in cosmological models with the same normalisation of the power spectrum. Indeed, in this case, we are comparing the same clusters in different cosmological models, and thus
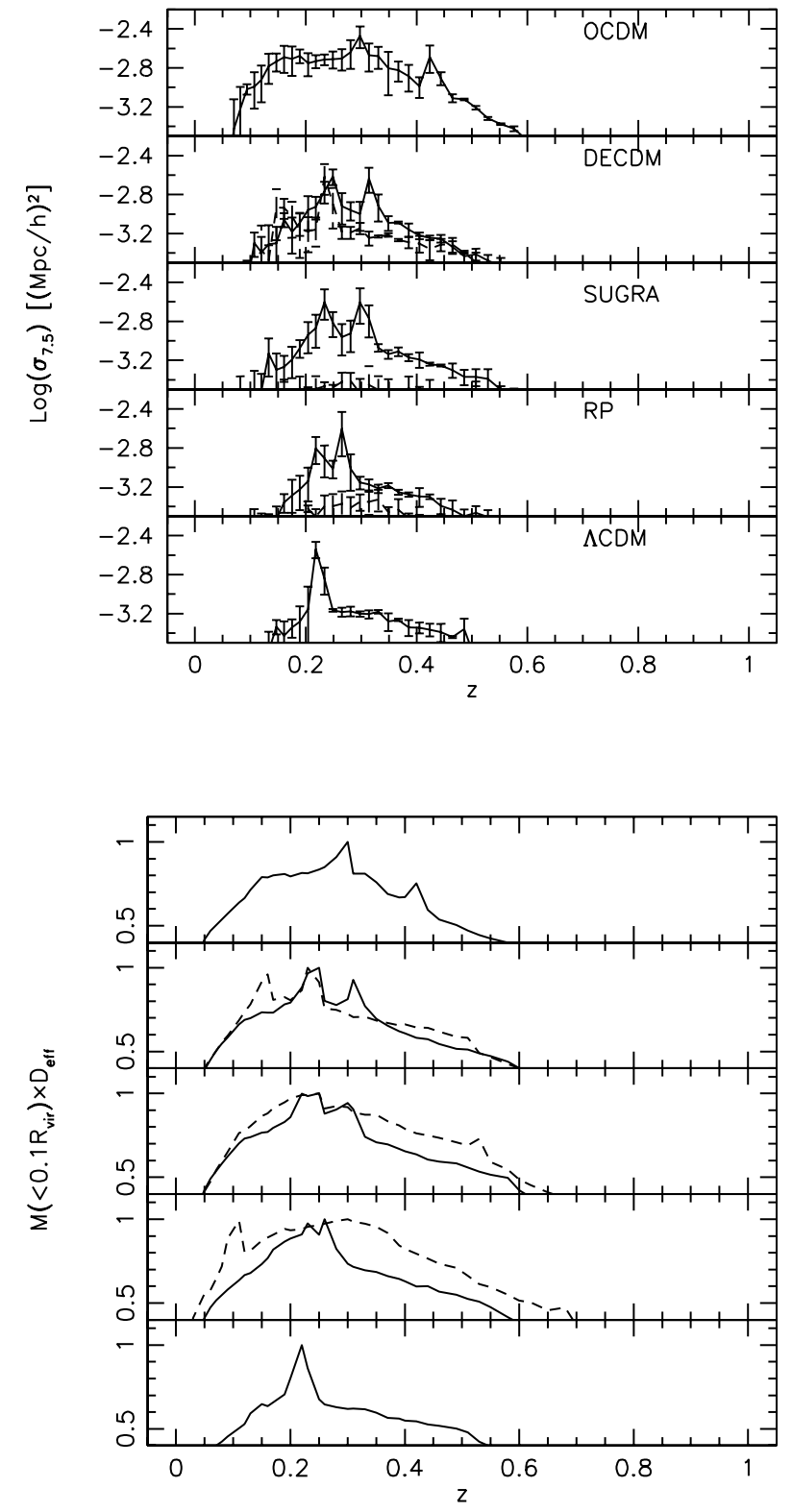

Fig. 4. Top panel: lensing cross sections for arcs with length-to-width ratio larger than 7.5 of one of the most massive clusters in our sample as a function of the lens redshift in different cosmological models. As in Fig. 3, sources are kept at redshift $z_{\mathrm{s}}=1$. Solid curves show the results for cosmologies normalised to $\sigma_{8}=0.9$. Dashed curves show the corresponding results for $\sigma_{8}$ reduced according to the CMB normalisation. The error bars show the variance among the three cluster projections. Bottom panel: redshift evolution of the mass enclosed by cluster-centric spheres having $10 \%$ of the virial radius, multiplied by the effective lensing distance, for the same cluster as in the top panel. The curves are arbitrarily normalised to reach unity at maximum. Solid and dashed lines refer to the different normalisations of the power spectrum. Evidently, peaks in the strong-lensing cross section coincide with the inflow of mass into the cluster centre, i.e. with merger events.

the same dynamical processes cause the cluster lensing efficiencies to peak. However, the position and the amplitude of the peaks depends strongly on the cosmological model. This 
becomes clearer by looking at the lensing cross sections of one individual cluster.

In the top panel of Fig. 4, we show the lensing cross section for arcs with length-to-width ratio larger than 7.5 of one of the most massive clusters in our sample $\left(\sim 10^{15} h^{-1} M_{\odot}\right)$. The error bars show the variance among its three projections. Solid curves refer to models with $\sigma_{8}=0.9$. Since the cluster forms at different epochs in different cosmological models, corresponding dynamical events during its formation occur at different redshifts, and consequently the cross-section peaks are shifted in time. Initial conditions of our clusters are set up such that single halos reach the same evolutionary state at redshift zero in all cosmological models. Thus, the time difference between dynamical events in different cosmologies shrinks as the redshift decreases.

The bottom panel of Fig. 4 shows the total mass enclosed by cluster-centric spheres having $10 \%$ of the virial radius. The virial radius is defined here as the radius enclosing an average density of 200 times the mean cosmic background density. For easier comparison with the cross sections in the top panel, the mass is multiplied with the effective lensing distance, such as to suppress the curves at redshifts where lensing is inefficient.

Sudden peaks in the curves indicate that large clumps of matter are passing through the core cluster region. As discussed in Torri et al. (2004), the typical time-scale for an infalling substructure to reach the peri-centre of its orbit is $\sim 1$ Gyr (Tormen et al. 2004). All the pronounced peaks in the curves representing the lensing cross sections evidently correspond to comparably pronounced peaks in the cluster core mass, which demonstrates our previous assertion on the relation between merger events and maxima in the cluster lensing efficiency. A similar analysis performed on the other clusters in our sample confirmed that all the highest peaks in the lensing cross sections occur during merger events. The curves in the lower panel of Fig. 4 also show that even quite moderate changes of the cluster core mass can cause substantial changes in the arc cross sections.

Had we not multiplied the mass by the effective lensing distance, mass peaks at high and low redshift would occurr wich do not correspond to peaks in the lensing cross sections, merely reflecting the geometrical sensitivity of lensing.

It is remarkable that even if the lens is intrinsically not very efficient for lensing, the passage of massive substructures through the cluster centre can strongly enhance its ability for producing gravitational arcs. For example, in the $\Lambda$ CDM model, the lensing cross section is larger by roughly one order of magnitude while a merger is occurring. Similar increments in the amplitude of the lensing cross section have been found by Torri et al. (2004). This suggests that at least a fair fraction of strong-lensing events in galaxy clusters may be transient events, characterising peculiar epochs during cluster evolution.

When the CMB normalisation of the power spectrum is adopted, the comparison between the same clusters in different cosmological models is less straightforward. Indeed, changing $\sigma_{8}$ strongly affects the merger history of the cluster, as shown by the dotted lines in Fig. 4.

We also note that in both the RP and the SUGRA models with CMB normalisation, even when the cluster appears to be dynamically perturbed, the lens still does not become particularly efficient for strong lensing. We verified that in these cases, the substructures producing the peaks in the velocity dispersion are orbiting at large distance from the cluster centre. Indeed, the merger events producing the peaks in the lensing cross sections in the cluster-normalised models are here delayed in time due to the reduced $\sigma_{8}$.

\subsection{Optical depth}

We can now estimate the probability for arc formation by a population of clusters. Given a mass function, $\mathrm{d} n(M, z) / \mathrm{d} z$, assuming that the typical lensing cross sections for lenses of mass $M$ are well represented by the lensing cross sections of our sample $\sigma(M, z)$, the optical depth $\tau\left(z_{\mathrm{s}}\right)$ is defined as the sum of the lensing cross sections of all lenses between the observer and the sources, divided by the area of the source plane. Following this definition,

$\tau\left(z_{\mathrm{s}}\right)=\frac{1}{4 \pi D_{\mathrm{s}}^{2}} \int_{0}^{z_{\mathrm{s}}} \mathrm{d} z(1+z)^{3}\left|\frac{\mathrm{d} V}{\mathrm{~d} z}\right| \int_{0}^{\infty} \mathrm{d} M \frac{\mathrm{d} n}{\mathrm{~d} M} \sigma(M, z)$,

where $z_{\mathrm{s}}$ is the source redshift, $V$ is the cosmic volume, and the factor $(1+z)^{3}$ accounts for the fact that the cluster number density is defined per comoving volume. The former equation applies under the assumption that lensing cross sections do not overlap, i.e. that the total optical depth is low, $\tau \ll 1$.

Since we do not know $\sigma(M, z)$ for a continuous range of masses, but only for a discrete cluster sample with masses $M_{i}$, we calculate the optical depth as follows:

$$
\begin{aligned}
\tau\left(z_{\mathrm{s}}\right)= & \frac{1}{4 \pi D_{\mathrm{s}}^{2}} \int_{0}^{z_{\mathrm{s}}} \mathrm{d} z(1+z)^{3}\left|\frac{\mathrm{d} V}{\mathrm{~d} z}\right| \\
& \times \sum_{i=1}^{n-1} \frac{1}{2}\left[\sigma\left(M_{i}, z\right)+\sigma\left(M_{i+1}, z\right)\right] \\
& \times \int_{M_{i}}^{M_{i+1}} \frac{\mathrm{d} n(M, z)}{\mathrm{d} M} \mathrm{~d} M
\end{aligned}
$$

where $n$ is the total number of clusters in our sample. This corresponds to attaching to each halo of mass $M$ in the interval $\left[M_{i}, M_{i+1}\right]$ a lensing cross section $\sigma(M, z)=1 / 2\left[\sigma\left(M_{i}, z\right)+\right.$ $\left.\sigma\left(M_{i+1}, z\right)\right]$. We use here the Sheth-Tormen mass function (Sheth \& Tormen 1999), which was shown to fit well the results of numerical simulations in several dark-energy cosmologies (Klypin et al. 2003).

The integrand in Eq. (7) is shown for different cosmologies in Fig. 5. The bootstrap error bars illustrate the uncertainty due to different possible orientations of each individual lens. The differential optical depth for arcs with length-to-width ratio larger than 7.5 and for sources at redshift $z_{\mathrm{s}}=1$ is larger in those cosmological models where lenses form earlier and are thus more concentrated. Moreover, the contribution to the total optical depth comes from clusters in a wider redshift range in these cosmologies. For example, in the high redshift tail, the curves drop to zero at $z \sim 0.65$ and $z \sim 0.8$ in the $\Lambda \mathrm{CDM}$ model and in the OCDM models, respectively. Moreover, at $z \sim 0.6$, the differential optical depth is still close to its maximum in the OCDM model, while it is decreased below $30 \%$ in 


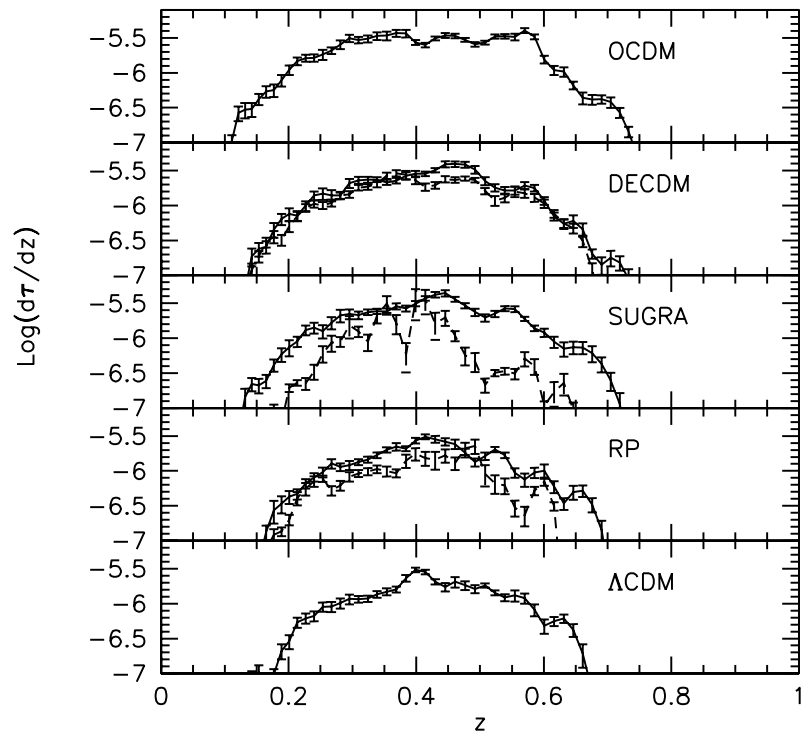

Fig. 5. Differential optical depth for arcs with $L / W>7.5$ and sources at $z_{\mathrm{s}}=1$ as a function of the lens redshift. Solid curves show the results for cosmologies with $\sigma_{8}=0.9$. Dashed curves show the corresponding results when $\sigma_{8}$ is reduced according to the CMB normalisation.

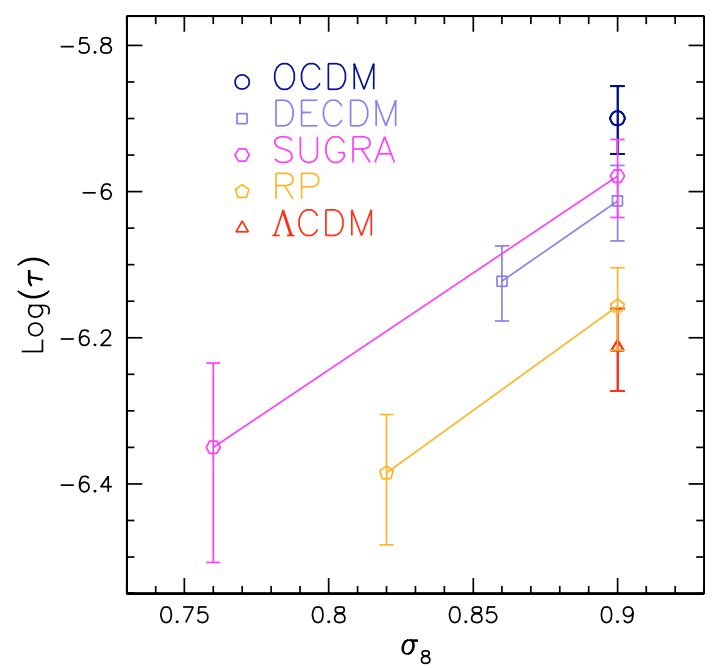

Fig. 6. Optical depth for arcs with $L / W>7.5$ and sources at $z_{\mathrm{s}}=1$ as a function of the normalisation of the power spectrum.

the $\Lambda$ CDM. Other cosmologies, like the RP, the DECDM and the SUGRA with cluster-abundance normalisation, interpolate between these models, while we obtain substantially smaller optical depths by adopting the CMB normalisation of the power spectrum.

The total optical depth as a function of the normalisation of the power spectrum is shown in Fig. 6. Again, we find that, when cluster normalisation is used, dark-energy models interpolate between $\Lambda$ CDM and OCDM. When the normalisation is reduced according to the amplitude of large-scale temperature fluctuations in the $\mathrm{CMB}$, the optical depth also decreases as shown by the solid lines in the figure.

The total optical depth changes by a factor of two between the $\triangle \mathrm{CDM}$ and the OCDM models. This is in disagreement with the previous results of Bartelmann et al. (1998), where one

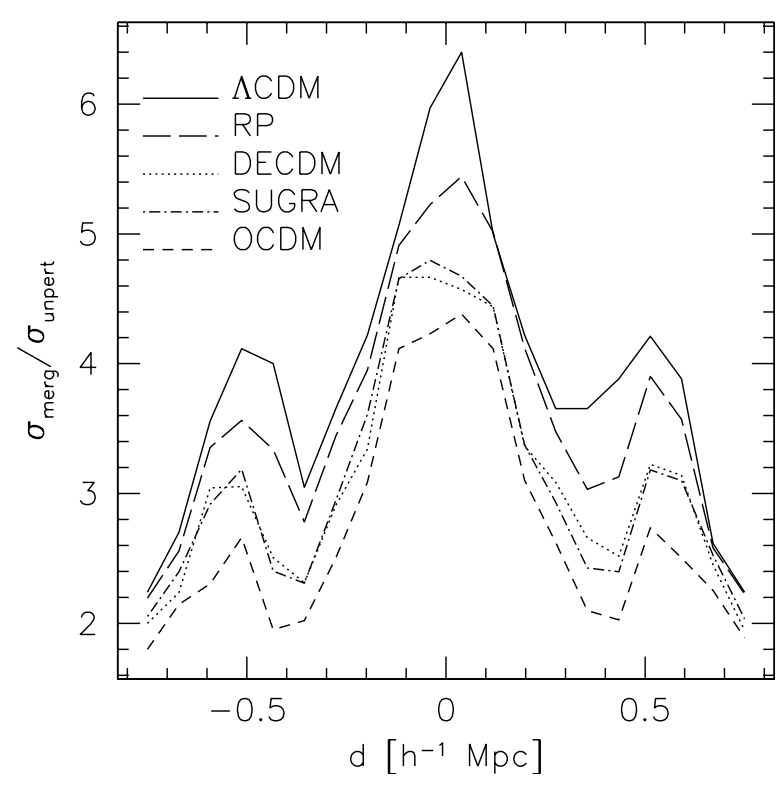

Fig. 7. Cross sections for arcs with $L / W>7.5$ as a function of the distance between two merging halos, modelled using a pseudo-elliptical NFW lens model with ellipticity $e=0.3$. The first halo has a mass of $M_{\text {main }}=7.5 \times 10^{14} h^{-1} M_{\odot}$, the second has $M_{\text {sub }}=3 \times 10^{14} h^{-1} M_{\odot}$. The lens and source planes are placed at $z_{1}=0.3$ and $z_{\mathrm{s}}=2$, respectively. Different line types refer to different cosmological models.

order of magnitude larger optical depths were found in OCDM compared to $\Lambda$ CDM. However, we use here a modified method for estimating the optical depth. Moreover, both the mass and in particular the time resolutions of our simulations are considerably larger than in the earlier paper. This allows us to better account for the contribution to the optical depth from all the merger events occurring in the lenses, all of which enhance the cluster efficiency for producing arcs, as shown in the previous sections. The impact of mergers appears to be different in different cosmological models, being larger in those cosmologies where lenses are less concentrated. As a consequence of that, the difference in the number of arcs which is expected to be observed in $\Lambda \mathrm{CDM}$ and in OCDM cosmological models is reduced when mergers are accounted for. Indeed, resampling our cross sections for reproducing the same time resolution of Bartelmann et al. (1998), we obtain an optical depth which is lower by a factor of eight in the $\Lambda \mathrm{CDM}$ compared to the OCDM model, in agreement with Bartelmann et al. (1998).

This interpretation is also supported by the following simple experiment. We simulate the passage of a clump of matter of mass $M_{\text {sub }}=3 \times 10^{14} h^{-1} M_{\odot}$ through the centre of a halo of mass $M_{\text {main }}=7.5 \times 10^{14} h^{-1} M_{\odot}$. Both the main cluster clump and the merging substructure are modelled using the pseudo-elliptical Navarro-Frenk-White lens model discussed by Meneghetti et al. (2003b), assuming an ellipticity of the lensing-potential contours of $e=0.3$. The lens and source redshifts are kept fixed at $z_{1}=0.3$ and $z_{\mathrm{s}}=2$, respectively. Having placed the main halo at the centre of the lens plane, we produce deflection-angle maps for several distances between the merging clumps by moving the substructure along the $x$-axis. Then, we apply the methods discussed in the previous sections for determining how the lensing cross sections evolve as functions 


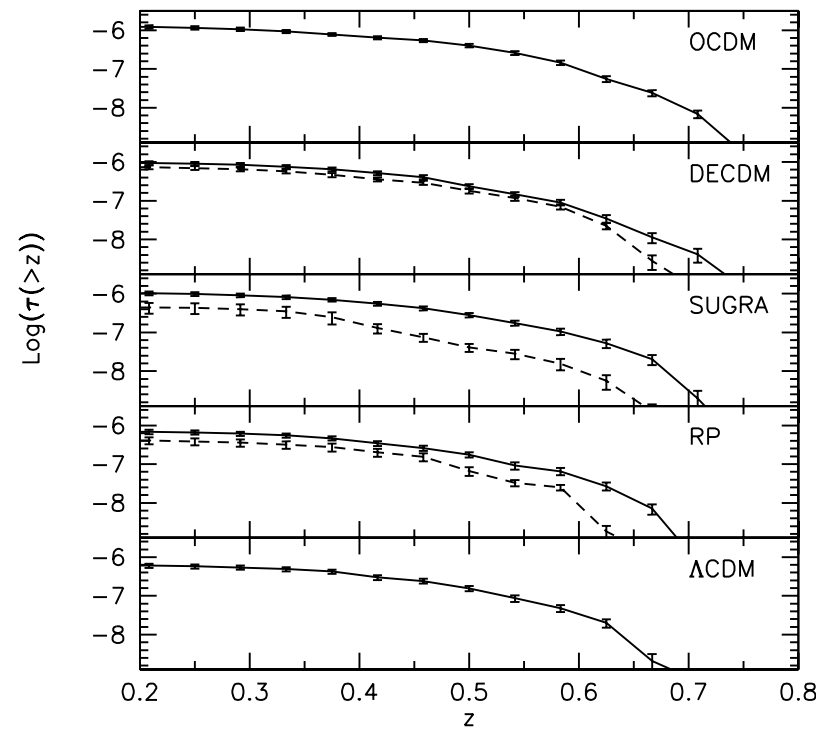

Fig. 8. Optical depth as a function of the minimal lens redshift contributing to the integral in Eq. (7). All cross sections of lenses below redshift $z$ were neglected for this cumulative plot.

of the distance between the colliding halos. Results are shown in Fig. 7. Different line types refer to different cosmological models and all curves are normalised to the cross section of an unperturbed halo of mass $=7.5 \times 10^{14} h^{-1} M_{\odot}$.

We note that in this analytic experiment we reproduce well the three peaks in the lensing cross sections which were previously found in numerical lensing simulations by Torri et al. (2004). As discussed there, the first and third peaks are due to the increasing shear in the region between the two merging halos, while the substructure approaches the main cluster clump. The second peak is due to the larger convergence of the lens when the two clumps are perfectly aligned. As anticipated earlier, the growth of the lensing cross sections relative to the unperturbed case is larger by more than $50 \%$ in the $\Lambda \mathrm{CDM}$ than in the OCDM model. Other cosmological models with static and dynamical dark energy interpolate between $\Lambda \mathrm{CDM}$ and OCDM, confirming our assertion that mergers have a stronger impact in cosmological models where halos are less concentrated.

Bartelmann et al. (2003) already estimated using analytic models the change of the optical depth relative to the $\Lambda \mathrm{CDM}$ model in different cosmological models with constant equation of state of the dark energy. The only cosmological model we investigated here, which can be directly compared to the results of Bartelmann et al. (2003) is the DECDM model with $\mathrm{CMB}$ normalisation. In this particular case, we find an optical depth which is larger by roughly $25 \%$ compared to the $\Lambda \mathrm{CDM}$ case, while the analytic models predicted a larger increment, i.e. between $50 \%$ and $90 \%$. This inconsistency is again due to the smaller impact of mergers in the DECDM compared to the $\Lambda$ CDM model.

As discussed earlier, we see larger differences in the differential optical depth among different cosmological models at high redshift. This is better shown in Fig. 8, where we plot the integrated optical depth as a function of redshift, when the contribution from lenses below redshift $z$ is neglected. The curves show that cluster lenses start contributing to the lensing optical depth at increasingly larger redshift in the dark energy cosmologies and in the OCDM compared to $\Lambda$ CDM model. Therefore, searching for arcs in high-redshift clusters can be very promising for constraining both the cluster evolution and the equation of state of dark energy.

\section{Summary}

We have investigated the efficiency of numerically simulated galaxy clusters for producing strong lensing events in different cosmological models with constant and time-variable equation of state of the dark energy, and we have compared it with "standard" $\Lambda \mathrm{CDM}$ and OCDM models. Both the local abundance of galaxy clusters and the observed amplitude of the temperature fluctuations in the CMB have been used for normalising the power spectrum of the primordial density perturbations generating our sample of numerically simulated clusters. In the latter case, the normalisation is reduced for accounting for the increasing ISW effect affecting in different ways the large-scales CMB anisotropies in the cosmologies we considered. When the local cluster-abundance normalisation is used, the initial conditions in the $N$-body simulations were set up such as to obtain halos having approximately the same evolution at redshift zero.

Our main results can be summarised as follows:

- The lensing cross sections for long and thin arcs averaged over all clusters in our sample reflect the differences in the concentration of dark matter halos in different cosmological models. Halos are more efficient for producing strong gravitational lensing events in those cosmological models where they are more concentrated. Assuming the cluster abundance normalisation of the power spectrum, we find lensing cross sections which are smaller by roughly a factor of four in the $\Lambda$ CDM compared to the OCDM model, and the other dark energy models interpolate between them. Moreover, despite the present equation of state being the same for the RP and the SUGRA models, their lensing cross sections differ substantially. For those dark energy models with CMB normalisation, we find lensing cross sections smaller by more than one order of magnitude compared to the OCDM model, indicating that the power spectrum normalisation is a crucial aspect for assessing the relative behaviour of the strong-lensing effect in different dark energy cosmologies.

- We find that the local maxima in the lensing efficiency are caused by merger events occurring in the lenses. In particular, we verify that the peaks in the redshift evolution of lensing cross sections of individual clusters are shifted in time due to the offset between the merger histories in different cosmologies.

- The optical depth for lensing is larger in those cosmological models where lenses form earlier and are thus more concentrated. Moreover, the contribution to the total optical depth comes from clusters in a wider redshift range in these cosmologies. 
- Compared to the $\Lambda \mathrm{CDM}$ model, the optical depth in the OCDM model is larger by only a factor of two, in disagreement with the results of earlier work, which found an order-of-magnitude difference between these two models. However, the better time resolution of our simulations allows us to account for the merger events occurring in the lenses, which we verified to have a stronger impact in those cosmological models where lenses are less concentrated.

- When the same normalisation of the power spectrum is adopted, we find that clusters in the dark-energy models we investigated have optical depths within the boundaries set by the $\Lambda$ CDM and the OCDM models. However, when the CMB normalisation is used, the optical depths become significantly smaller. The differences between the cosmological models are relatively small, but they become larger when the contribution to the lensing optical depths from high redshift clusters is considered.

Based on these results, we conclude that arc statistics is a potentially powerful tool for constraining the equation of state of dark energy, and for investigating the dynamical evolution of galaxy clusters, provided $\sigma_{8}$ is known to sufficient accuracy. In particular, strong lensing events at high redshift represent an important source of information which might be used for discriminating between dark energy models. Further investigations are needed now for fully understanding the impact of mergers on the lensing cross sections and its dependence on cosmology.

Acknowledgements. The lensing simulations were carried out on the IBM-SP4 machine at the "Rechenzentrum Garching der MaxPlanck-Gesellschaft und des IPP" (Garching). The $N$-body simulations were performed at the "Centro Interuniversitario del Nord-Est per il Calcolo Elettronico" (CINECA, Bologna), with CPU time assigned under an INAF-CINECA grant. K. Dolag acknowledges support by a Marie Curie Fellowship of the European Community program "Human Potential" under contract number MCFI-2001-01227. Carlo Baccigalupi and Francesca Perrotta were supported in part by the NASA LTSA grant NNG04GC90G.

\section{References}

Baccigalupi, C., Matarrese, S., \& Perrotta, F. 2000, PRD, 62, 123510 Baccigalupi, C., Balbi, A., Matarrese, S., Perrotta, F., \& Vittorio, N. 2002, PRD, 65, 063520

Bartelmann, M., \& Weiss, A. 1994, A\&A, 287, 1

Bartelmann, M., Steinmetz, M., \& Weiss, A. 1995, A\&A, 297, 1

Bartelmann, M., Huss, A., Colberg, J., Jenkins, A., \& Pearce, F. 1998, A\&A, 330, 1

Bartelmann, M., Perrotta, F., \& Baccigalupi, C. 2002, A\&A, 396, 21
Bartelmann, M., Meneghetti, M., Perrotta, F., Baccigalupi, C., \& Moscardini, L. 2003, A\&A, 409, 449

Bennett, C., Halpern, M., Hinshaw, G., et al. 2003, ApJS, 148, 1

Brax, P., \& Martin, J. 2000, PRD, 61, 103502

Dalal, N., Holder, G., \& Hennawi, J. F. 2004, ApJ, 609, 50

Dolag, K., Bartelmann, M., Perrotta, F., Baccigalupi, C., et al. 2004, A\&A, 416, 853

Gladders, M., Hoekstra, H., Yee, H., Hall, P., \& Barrientos, L. 2003, ApJ, 593, 48

Hasinger, G., Giacconi, R., Gunn, J., et al. 1998, A\&A, 340, L27

Hawkins, E., Maddox, S., Cole, S., Lahav, O., et al. 2003, MNRAS, 346,78

Hockney, R., \& Eastwood, J. 1988, Computer simulation using particles (Bristol: Hilger)

Jenkins, A., Frenk, C., White, S., et al. 2001, MNRAS, 321, 372

Klypin, A., Macciò, A., Mainini, R., \& Bonometto, S. 2003, ApJ, 599, 31

Lidman, C., Rosati, P., Demarco, R., et al. 2004, A\&A, 416, 829

Macciò, V. A., 2005, MNRAS, 361, 1250

Meneghetti, M., Bolzonella, M., Bartelmann, M., Moscardini, L., \& Tormen, G. 2000, MNRAS, 314, 338

Meneghetti, M., Yoshida, N., Bartelmann, M., et al. 2001, MNRAS, 325,435

Meneghetti, M., Bartelmann, M., \& Moscardini, L. 2003a, MNRAS, 346,67

Meneghetti, M., Bartelmann, M., \& Moscardini, L. 2003b, MNRAS, 340, 105

Oguri, M., Lee, J., \& Suto, Y. 2003, ApJ, 599, 7

Peebles, P., \& Ratra, B. 2002, Rev. Mod. Phys., 75, 599

Perlmutter, S., Aldering, G., Goldhaber, G., Knop, R., et al. 1999, ApJ, 517,565

Perrotta, F., \& Baccigalupi, C. 1999, PRD, 59, 123508

Perrotta, F., Baccigalupi, C., \& Matarrese, S. 2000, PRD, 61, 023507

Ratra, B., \& Peebles, P. 1988, PRD, 37, 3406

Riess, A., Strolger, L.-G., Tonry, J., Casertano, S., et al. 2004, ApJ, 607, 665

Riess, A. G., Filippenko, A. V., Challis, P., et al. 1998, AJ, 116, 1009

Seljak, U., \& Zaldarriaga, M. 1996, ApJ, 469, 437

Sheth, R., \& Tormen, G. 1999, MNRAS, 308, 119

Spergel, D., Verde, L., Peiris, H., Komatsu, E., et al. 2003, ApJS, 148, 175

Springel, V., Yoshida, N., \& White, S. 2001, New Astron., 6, 79

Tegmark, M., Strauss, M., Blanton, M., Abazajian, S., et al. 2004, PRD, 69, 103501

Thompson, D., Pozzetti, L., Hasinger, G., et al. 2001, A\&A, 377, 778

Tormen, G., Bouchet, F., \& White, S. 1997, MNRAS, 286, 865

Tormen, G., Moscardini, L., \& Yoshida, N. 2004, MNRAS, 350, 1397

Torri, E., Meneghetti, M., Bartelmann, M., et al. 2004, MNRAS, 349, 476

Wambsganss, J., Cen, R., \& Ostriker, J. 1998, ApJ, 494, 29

Wambsganss, J., Bode, P., \& Ostriker, J. 2004, ApJ, 606, L93

Weinberg, N., \& Kamionkowski, M. 2003, MNRAS, 341, 251

Wetterich, C. 1988, Nuclear Physics B, 302, 668

Yoshida, N., Sheth, R., \& Diaferio, A. 2001, MNRAS, 328, 669

Zaritsky, D., \& Gonzalez, A. 2003, ApJ, 584, 691 